\title{
1 Lack of signal for the impact of venom gene diversity on speciation 2 rates in cone snails
}

4 Mark A Phuong ${ }^{1}$, Michael E Alfaro ${ }^{1}$, Gusti N Mahardika ${ }^{2}$, Ristiyanti M Marwoto ${ }^{3}$, Romanus

5 Edy Prabowo ${ }^{4}$, Thomas von Rintelen ${ }^{5}$, Philipp WH Vogt ${ }^{5}$, Jonathan R Hendricks ${ }^{6}$, Nicolas

$6 \quad$ Puillandre ${ }^{7}$

7

$8{ }^{1}$ Department of Ecology and Evolutionary Biology, University of California, Los Angeles, CA 990095, USA

$10{ }^{2}$ Animal Biomedical and Molecular Biology Laboratory, Faculty of Veterinary Medicine,

11 Udayana University Bali, Jl Sesetan-Markisa 6, Denpasar, Bali 80225, Indonesia

$12{ }^{3}$ Zoology Division (Museum Zoologicum Bogoriense), Research Center for Biology, LIPI,

13 Cibinong, Bogor, Indonesia

$14 \quad{ }^{4}$ Faculty of Biology, Jenderal Soedirman University, Purwokerto, Indonesia

$15{ }^{5}$ Museum für Naturkunde - Leibniz Institute for Evolution and Biodiversity Science, Berlin, 16 Germany

$17 \quad{ }^{6}$ Paleontological Research Institution, Ithica, NY 14850, USA

$18{ }^{7}$ Institut Systématique Evolution Biodiversité (ISYEB), Muséum national d'Histoire naturelle,

19 CNRS, Sorbonne Université, EPHE, 57 rue Cuvier, CP 26, 75005 Paris, France

20

\section{Corresponding author:}

22 M. A. Phuong

23 E-mail: markphuong@gmail.com 


\section{Abstract}

26 Understanding why some groups of organisms are more diverse than others is a central goal in

27 macroevolution. Evolvability, or lineages' intrinsic capacity for evolutionary change, is thought

28 to influence disparities in species diversity across taxa. Over macroevolutionary time scales,

29 clades that exhibit high evolvability are expected to have higher speciation rates. Cone snails

30 (family: Conidae, >900 spp.) provide a unique opportunity to test this prediction because their

31 venom genes can be used to characterize differences in evolvability between clades. Cone snails

32 are carnivorous, use prey-specific venom (conotoxins) to capture prey, and the genes that encode

33 venom are known and diversify through gene duplication. Theory predicts that higher gene

34 diversity confers a greater potential to generate novel phenotypes for specialization and

35 adaptation. Therefore, if conotoxin gene diversity gives rise to varying levels of evolvability,

37 exon capture techniques to recover phylogenetic markers and conotoxin loci across 314 species,

38 the largest venom discovery effort in a single study. We paired a reconstructed timetree using 12

39 fossil calibrations with species-specific estimates of conotoxin gene diversity and used trait-

40 dependent diversification methods to test the impact of evolvability on diversification patterns.

41 Surprisingly, did not detect any signal for the relationship between conotoxin gene diversity and

42 speciation rates, suggesting that venom evolution may not be the rate-limiting factor controlling

43 diversification dynamics in Conidae. Comparative analyses showed some signal for the impact

44 of diet and larval dispersal strategy on diversification patterns, though whether or not we

45 detected a signal depended on the dataset and the method. If our results remain true with

46 increased sampling in future studies, they suggest that the rapid evolution of Conidae venom 
47 may cause other factors to become more critical to diversification, such as ecological opportunity or traits that promote isolation among lineages.

\section{Introduction}

51 Why are some taxa more diverse than others? Species richness and phenotypic diversity are not

52 distributed evenly across the tree of life (Rabosky et al. 2013). For example, there exists over

5310,000 species of birds, but their closest relatives (crocodiles and alligators) comprise only of 23

54 species. Differences in evolvability, or lineages' intrinsic capacity to adapt and diversify, is one

55 reason commonly used to explain these disparities (Wagner \& Altenberg 1996; Yang 2001;

56 Jones et al. 2007; Pigliucci 2008; Losos 2010). Evolvability is thought to be determined by the

57 underlying genetic architecture of organisms - some genomes of organisms have a greater

58 propensity to generate variation that may be adaptive in the future (Wagner \& Altenberg 1996;

59 Jones et al. 2007; Pigliucci 2008). For example, gene duplication increases evolvability - the

60 copied gene is free from the selective pressures of the original gene (Crow \& Wagner 2006).

61 Mutation, selection, and drift can act on the copied gene, facilitating the possibility of new

62 phenotypes to arise; this shapes the extent that taxa can diversify and exploit resources (Crow \&

63 Wagner 2006). Over long evolutionary time scales, clades that exhibit higher evolvability are

64 predicted to have increased species richness and diversification rates (Yang 2001).

67 phenotype (Hoekstra \& Coyne 2007). Past studies that have attempted to test the impact of 
et al. 2016). For example, whole genome duplication events, which are hypothesized to increase

71 the genomic potential of organisms, have been documented to increase (Santini et al. 2009;

72 Soltis et al. 2009; Tank et al. 2015), decrease (Mayrose et al. 2011), and have no impact (Zhan et

73 al. 2014) on the long-term evolutionary success of clades. In another case, a positive correlation

74 between evolvability and speciation rates exist when measuring evolvability through

75 morphological proxies (Rabosky et al. 2013). One limitation of past research on this hypothesis

76 is the inability to tie genomic changes with ecological factors driving diversification patterns

77 (Robertson et al. 2017). Although gene duplication and whole genome duplication events can

78 increase the evolutionary capacity of organisms, genes that are ecologically relevant for

79 adaptation may not be readily available for selection to drive divergence.

Here, we study the relationship between evolvability and diversification in cone snails

81 (family, Conidae), a diverse group (> 900 spp.) of predatory marine gastropods. These snails

82 feed on either worms, molluscs, or fish by paralyzing their prey with a cocktail of venomous

83 neurotoxins (conotoxins, Duda \& Palumbi 1999). Cone snail provides a unique opportunity to

84 test predictions of evolvability and diversification for the following reasons: first, cone snail

85 species share an ecologically relevant trait, venom. Conidae species are globally distributed in

86 tropical and subtropical regions, where $>30$ species can co-occur within the same habitat (Kohn

87 2001). High numbers of species hypothesized to be able to co-occur because species have

88 diversified to specialize on different prey using prey-specific conotoxins (Duda \& Palumbi

89 1999). Second, venom genes are known and diversify through gene duplication (Duda \&

90 Palumbi 2000; Kaas et al. 2010, 2012; Chang \& Duda 2012). Diet specialization is thought to be

91 enabled by the rapid evolution of the genes that underlie conotoxins - estimated rates of gene

92 duplication and nonsynonymous substitutions rates for conotoxin genes are the highest across 
metazoans (Duda \& Palumbi 2000; Chang \& Duda 2012). Therefore, conotoxin genes provide a natural way to characterize differences in evolvability between clades.

We employ a sequence capture technique previously used in cone snails (Phuong \& Mahardika 2017) to recover phylogenetic markers and conotoxin genes from 314 described species. We use the phylogenetic markers to reconstruct a time-calibrated phylogeny and perform trait-dependent diversification analyses to test the impact of evolvability on diversification patterns. We predict that clades with a greater number of conotoxin gene copies should have higher speciation rates. In addition, we test other traits that may have an impact on diversification patterns, including diet and larval dispersal strategy.

\section{Methods}

\section{Bait design}

We used a targeted sequencing approach to recover markers for phylogenetic inference and obtain an estimate of conotoxin gene diversity from Conidae species. For the phylogenetic markers, we identified loci using a previous Conidae targeted sequencing dataset (Phuong \& Mahardika 2017) and the Conidae transcriptome data from (Phuong et al. 2016). In the Conidae targeted sequencing dataset, the authors generated a phylogeny using 5883 loci across 32 species (Phuong \& Mahardika 2017). For our sequencing experiment, we only retained loci that were $>180$ bp and were present in at least 26 out of 32 taxa with at least $10 \mathrm{X}$ coverage. We chose to only include longer loci to increase confidence in identifying orthologous fragments in other Conidae species. To identify additional phylogenetic markers from the transcriptome data 
115 (Phuong et al. 2016), which consisted of venom duct transcriptomes from 12 species, we

116

117

118

119

120

121

122

123

124

125

126

127

128

129

130

131

132

133

134

135

136

137 performed the following:

(1) identified reciprocal best blast hits between the assembled transcriptome and the Lottia gigantea protein reference (Simakov et al. 2013) using BLAST+ v2.2.31 (evalue = 1e-10). We also considered fragments that had their best hit to the protein reference, but to a non-overlapping portion ( $<20 \%$ overlapping).

(2) mapped reads using bowtie2 v2.2.7 (Langmead \& Salzberg 2012)

(3) removed duplicates using picard-tools v.2.1.1 (http://broadinstitute.github.io/picard)

(4) fixed assembly errors by calling single nucleotide polymorphisms (SNPs) using samtools v1.3 and bcftools v1.3 (Li et al. 2009)

(5) aligned sequences per locus using mafft v7.222 (Katoh et al. 2005)

(6) calculated uncorrected pairwise distances within each locus for all possible pairwise comparisons

(7) removed sequences if the uncorrected pairwise distance was greater than the $90^{\text {th }}$ percentile for those pair of species

(8) denoted exon boundaries by comparing the transcriptome sequences to the Lottia gigantea genome reference (Simakov et al. 2013), retaining exons >180bp

For all retained phylogenetic markers, we also performed the following: (1) we generated an ancestral sequence using FastML v3.1 (Ashkenazy et al. 2012) between a Californiconus

californicus sequence and another Conidae sequence that had the highest amount of overlap with the $C$. californicus sequence (we generated these ancestral sequences to decrease the genetic distances between the target sequence and the orthologous sequence from any Conidae species), (2) removed sequences that had a GC content $<30 \%$ or $>70 \%$ because extreme GC contents can 
138

139

140

141

142

143

144

145

146

147

148

149

150

151

152

153

154

155

156

157

158

159

160

reduce capture efficiency (Bi et al. 2012), (3) removed loci that contained repeats identified through the RepeatMasker v4.0.6 web server (Smit et al. 2015), and (4) performed a self-blast with the target sequences via blastn v2.2.31 (evalue $=1 \mathrm{e}-10)$ and removed loci that did not blast to itself with sequence identity $>90 \%$. The final set of target loci for phylogenetic inference included 1749 loci, with a total length of 470,435 bp.

To recover conotoxin loci, we targeted sequences generated from both the previous targeted sequencing dataset (Phuong \& Mahardika 2017) and the transcriptome dataset (Phuong et al. 2016). For conotoxin sequences discovered from the targeted sequencing dataset (Phuong \& Mahardika 2017), we performed the following to generate our target sequences: (1) we trimmed each sequence to only retain the coding region and included 100bp flanking the exon, (2) merged sequences using cd-hit v4.6.4 (Li \& Godzik 2006) at 95\% sequence similarity to reduce redundancy among conotoxin loci (3) masked repeats using the RepeatMasker v4.0.6 web server (Smit et al. 2015), and (4) retained loci >120bp to ensure that the locus was longer than our desired bait sequence length. We concatenated all sequences below $120 \mathrm{bp}$ to create a single, chimeric sequence for capture. The final set of target sequences from the previous targeted sequencing dataset consisted of 12,652 unique loci totaling 3,113,904 bp and a single concatenated sequence representing 351 merged loci with a total length of 37,936 bp. We also targeted conotoxin loci from the transcriptomes described in (Phuong et al. 2016) to obtain conotoxin loci from gene superfamilies that were not targeted in (Phuong \& Mahardika 2017) or performed poorly. We performed the following to generate a set of conotoxin loci from the transcriptome data: (1) we trimmed sequences from (Phuong et al. 2016) to only include the coding region and 100bp of the untranslated regions (UTRs), (2) merged sequences using cd-hit v4.6.4 (Li \& Godzik 2006) at 97\% sequence similarity to reduce redundancy among conotoxin 
161 loci, and (3) masked repeats using the RepeatMasker v4.0.6 web server (Smit et al. 2015). This

162

163

164

165

166

167

168

169

170

171

172

173

174

175

176

177

178

179

180

181

182

183

filtered dataset contained 395 conotoxin loci with a total length of 171,317 bp.

We submitted the following datasets to MYcroarray (Ann Arbor, Michigan, USA) for

bait synthesis: (1) 1749 loci for phylogenetic inference, (2) 12652 conotoxin loci using data from

(Phuong \& Mahardika 2017), (3) a single concatenated sequence using data from (Phuong \&

Mahardika 2017), and (4) 395 additional conotoxin loci using transcriptome data from (Phuong

et al. 2016). We chose to synthesize a MYbaits-3 kit, which included 60,000 bait sequences to

accommodate all the targeted loci. Because our aim was to recover sequences from species

throughout Conidae, each bait sequence was 120bp in length, which increases the efficiency of

recovering divergent fragments. We used a $2 X$ tiling density strategy (a new probe every 60bp)

across the sequences from datasets (1) and (2) and used a $4 \mathrm{X}$ tiling density strategy (a new probe

every 30bp) across datasets (3) and (4). We chose to increase the tiling density for datasets (3)

and (4) because the boundaries between exons were not denoted and we wanted to ensure

effective capture of the conotoxin loci. The set of probe sequences will be made available on

DRYAD following publication.

\section{Genetic samples, library preparation, hybridization, and sequencing}

We performed the targeted sequencing experiment across 362 samples representing both described Conidae species and unique lineages/potential new species identified during routine species verification using the mitochondrial locus (results not shown), CO1 (Table S1, Folmer et al. 1994). We also sequenced Bathyoma sp. as an outgroup based on a recent molecular phylogeny of the Conoideans, a clade of gastropods that includes Conidae (Table S1, Puillandre et al. 2011). We obtained these genetic samples from two field expeditions in Indonesia and 
Australia and from five museum collections (Table S1). We extracted DNA from tissue using the EZNA Mollusc DNA kit (Omega Bio-Tek, Doraville, GA, USA). There was slight variation in tissue preservation strategy among samples, with most tissues preserved directly in $95 \%$ ethanol (Table S1). For 10 samples, tissue was not available but DNA was available from a previous extraction. For these samples, we ran the DNA through the EZNA Mollusc DNA kit to purify the DNA prior to library preparation. We extracted a minimum of $2000 \mathrm{ng}$ per sample prior to library preparation, when possible. We sheared DNA using a Biorupter UCD-200 (Diagenode) when necessary and used a $1 \mathrm{X}$ bead purification protocol to ensure that the DNA fragments per sample ranged from $250-600 \mathrm{bp}$, centered on $\sim 350 \mathrm{bp}$. We aimed to generate libraries with longer fragment sizes to ensure that we could recover exons containing the mature toxin region, which are often only recoverable because they are flanking conserved regions that are targeted by our bait design (Phuong \& Mahardika 2017).

We prepared libraries for following the (Meyer \& Kircher 2010) protocol with the following modifications: (1) we started library preparation with at least 2000ng, rather than the 500ng suggested by the protocol to increase downstream capture efficiency, (2) we performed 1X bead clean-up for all enzymatic reactions and (3) generated dual-indexed libraries by incorporating adapters with unique $7 \mathrm{bp}$ barcodes. We were able to re-use libraries for the 32 species sequenced in (Phuong \& Mahardika 2017) and incorporated new indexes for these samples.

We generated equimolar pools of 8 samples and hybridized probes with 2000ng of the pooled DNA for $\sim 24$ hours. We substituted the adapter blocking oligonucleotides provided by MYcroarray with custome xGen blocking oligonucleotides (Integrated DNA technologies). We performed 3 independent post-capture amplifications using 12 PCR cycles and pooled these 
207

208

209

210

211

212

213

214

215

216

217

218

219

220

221

222

223

224

225

226

227

228

229

products. We sequenced all samples across 5 Illumina HiSeq 4000 lanes with 100bp paired-end reads. We multiplexed 80 samples per lane for the first 4 lanes and multiplexed the remaining 43 samples on the last lane. Sequencing was carried out at the Vincent J. Coates Genomics

Sequencing Laboratory at UC Berkeley. We note that our third lane containing 80 samples was contaminated, with $65 \%$ of the reads belonging to corn DNA. We were able to resequence this entire lane, resulting in overall increased sequencing effort for samples belonging to our third lane (Table 1).

\section{Data filtration and initial assembly}

We filtered the raw read data as follows:

(1) we trimmed reads using Trimmomatic v0.36 under the following conditions: (a) we used the ILLUMINACLIP option to trim adapters with a seed mismatch threshold of 2, a palindrome clip threshold of 40, and a simple clip threshold of 15 , (b) we performed quality trimming used the SLIDINGWINDOW option with a window size of 4 and a quality threshold of 20, (c) we removed reads below 36bp by setting the MINLEN option to 36, and (d) we removed leading and trailing bases under a quality threshold of 15 . (2) we merged reads using FLASH v1.2.11 (Magoč \& Salzberg 2011) with a min overlap parameter of 5, a max overlap parameter of 100, and a mismatch ratio of 0.05 .

(3) we removed low complexity reads using prinseq v0.20.4 (Schmieder \& Edwards 2011) using the entropy method with a conservative threshold of 60.

We assembled the filtered read data using SPAdes v3.8.1 using default parameters and reduced redundancy in the resultant assemblies with cap3 (Huang \& Madan 1999) under default parameters and cd-hit v4.6 (Li \& Godzik 2006, sequence identity threshold =99\%). 
Phylogenetic data processing and filtering

To associate assembled contigs with the target sequences for phylogenetic inference, we

used blastn v2.2.31 (word size $=11$, evalue $=1 \mathrm{e}-10)$. For the set of target sequences that

EXONERATE v2.2.0 (Slater \& Birney 2005) using the est2genome model because we found

mapped reads using bowtie2 (very sensitive local and no discordant options enabled) to a

242 all contigs within a sample. We removed sequences if a contig had a heterozygosity value greater than two standard deviations away from the mean.

Conotoxin assembly, processing, and filtering

249 first mapped reads back to our assembled contigs using the 'very sensitive local' and no

250 discordant' options. Then, we identified conotoxins within our dataset by using blastn v2.2.31

251 (word size $=11$, evalue $=1 \mathrm{e}-10)$ to associate our assembled contigs (from SPAdes) with

252 conotoxins we targeted in the bait design. We generated a set of unique conotoxin 'seed 
253 sequences' (a short stretch $[\sim 100 \mathrm{bp}]$ of conotoxin-blasted sequence) using a combination of of

254 the pysam module (https://github.com/pysam-developers/pysam), cd-hit v4.6 (percent identity = 98\%), cap3 (overlap percent identity cutoff $=99 \%)$, blastn v2.2.31 (word size $=11$, evalue $=1 \mathrm{e}-$

256 10), and Tandem Repeats Finder v4.09 (Benson 1999, minscore = 12, maxperiod $=2$ ). We

257 mapped reads to these seed sequences using bowtie 2 v2.2.6 (very sensitive local and no

258 discordant options enabled) and built out the conotoxin sequences using the PRICE v1.2

259 algorithm, which uses an iterative mapping and extension strategy to build out contigs from

260 initial seed sequences (Ruby et al. 2013). We ran price on each seed sequence at 5 minimum

261 percentage identity (MPI) values $(90 \%, 92 \%, 94 \%, 96 \%, 98 \%)$ with a minimum overlap length

262 value of 40 and a threshold value of 20 for scaling overlap for contig-edge assemblies. A

263 reassembled sequence was retained if it shared $90 \%$ identity with the original seed sequence and

264 we reduced redundancy by only retaining the longest sequence per seed sequence out of the 5

265 MPI assembly iterations. This approach is described in further detail in (Phuong \& Mahardika

266 2017). We note that the final conotoxin sequences per sample consisted of exon fragments,

267 where each sequence represents a single conotoxin exon flanked by any adjacent noncoding

268 region.

We updated our conotoxin reference database because we targeted additional conotoxin

270 transcripts from (Phuong et al. 2016). We used blastn v2.2.31 (word size $=11$, evalue $=1 \mathrm{e}-10)$

271 and EXONERATE v2.2.0 to define exon/intron boundaries for these additional conotoxin

272 transcripts and added them to our conotoxin reference database. The final conotoxin reference

273 database consisted of conotoxin sequences with the coding regions denoted and gene superfamily

274 annotated. We also annotated the conotoxin sequences for functional region (e.g., signal, pre, 
275 mature, post) using blastn v2.2.31 (word size $=11$, evalue $=1 \mathrm{e}-10$ ) with a conotoxin reference

276 database that was previously categorized by functional region (Phuong \& Mahardika 2017).

With the final conotoxin reference database, we performed blastn v2.2.31 (word size =

27811 , evalue $=1 \mathrm{e}-10)$ searches between the conotoxin reference and every sample's re-assembled

279

280

281

282

283

284

285

286

287

288

289

290

291

292

293

294

295

296

297 conotoxin sequences. We retained sequences if they could align across the entire coding region

of the reference sequence. We guessed the coding region for each retained sequence by aligning

the query sequence with the reference conotoxin using mafft v7.222 and denoting the coding

region as the region of overlap with the exon in the reference conotoxin. We fixed misassemblies

by mapping reads with bowtie2 (very sensitive local and no discordant options enabled, score

$\min =\mathrm{L}, 70,1)$ back to each conotoxin assembly and marked duplicates using picard-tools

v2.0.1. We masked regions below $5 \mathrm{X}$ coverage and discarded sequences if coverage was below

$5 \mathrm{X}$ across the entire predicted coding region. To generate the final set of conotoxin sequences

per sample, we merged sequences using cd-hit v4.6.4 (percent identity $=98 \%$, use local sequence identity, alignment coverage of longer sequence $=10 \%$, alignment coverage of short sequence $=$ $50 \%)$.

\section{Targeted sequencing experiment evaluation}

We generated the following statistics to evaluate the overall efficiency of the capture experiment: (1) we calculated the \% reads mapped to our targets by mapping reads to a reference containing all targets (both phylogenetic markers and conotoxin sequences) using bowtie2 v2.2.7 (very sensitive local and no discordant options enabled, score $\min =\mathrm{L}, 70,1$ ), (2) we calculated the $\%$ duplicates that were identified through the picard-tools, and (3) we calculated average coverage across the phylogenetic markers and conotoxin sequences. We also evaluated the effect 
298 of tissue quality (measured by the maximum fragment length of the extracted DNA sample via

299 gel electrophoresis) and genus (only on Conus, Profundiconus, and Conasprella, the three genera

300 with more than 1 sample included in this study) on these capture efficiency metrics using an

301 Analysis of Variance (ANOVA). To assess the effectiveness of conotoxin sequence recovery, we

302 compared our capture results with conotoxin diversity estimates from (Phuong \& Mahardika

303 2017) and calculated the average change in those estimates.

Phylogenetic inference

In addition to the 362 samples that we sequenced in this study, we obtained sequences for

were filtered under conditions similar to the filtering strategy applied to the phylogenetic

312 (Safavi-Hemami et al. unpublished). With these transcriptomes, we trimmed data using

313 trimmomatic v0.36 and merged reads using flash using parameters previously described above.

314 We assembled each transcriptome using Trinity v2.1.1 (Grabherr et al. 2011) reduced

315 redundancy in these transcriptomes with cap3 and cd-hit (percent identity $=99 \%)$. We used

316 blastn (word size $=11$, evalue $=1 \mathrm{e}-10)$ to associate contigs with the phylogenetic markers present

317 in our dataset. We used bowtie2 v2.2.7 (very sensitive local and no discordant enabled), samtools

$318 \mathrm{v} 1.3$, and bcftools 1.3 to map reads and call SNPs. We removed sequences if they were below

$3194 \mathrm{X}$ coverage for $>30 \%$ of the sequence and masked bases if they were below $4 \mathrm{X}$ coverage. We 
321 the mean heterozygosity within a sample. We used to mafft v7.222 to align loci across a total of

322373 samples.

We inferred phylogenies under both maximum likelihood (Stamatakis 2006) and

324 coalescent-based methods (Mirarab \& Warnow 2015). We used RAxML v8.2.9 (Stamatakis

325 2006) to generate a maximum likelihood phylogeny using a concatenated alignment under a

326 GTRGAMMA model of sequence evolution and estimated nodal support via bootstrapping. We

327 generated the coalescent-based phylogeny using ASTRAL-II v5.5.9 (Mirarab \& Warnow 2015)

328 with individual locus trees generated under default parameters in RAxML v8.2.9. We estimated

329 local posterior probabilities as a measure of branch support (Sayyari \& Mirarab 2017). Due to

330 the underperformance of the capture experiment, we ran both phylogenetic analyses with loci

331 that had $80 \%$ of the taxa, $50 \%$ of the taxa, and $20 \%$ of the taxa. For each iteration, we removed

332 taxa that had $>90 \%$ missing data.

Time calibration

We estimated divergence times using a Bayesian approach with MCMCTree

implemented in PAML v4.9g (Yang 2007). Given the size of our alignments, we first estimated

337 branch lengths using baseml and then estimated divergence times using Markov chain Monte

338 Carlo (MCMC). We used a HKY85 $+\Gamma$ substitution model and used an independent rates clock

339 model. We left all other settings on default. We performed two independent runs of the analysis

340 and checked for convergence among the runs. To account for uncertainty in branching order in

341 our phylogeny, we executed dating analyses across all trees generated from RAxML.

For time calibration, we applied a maximum constraint of 55 million years at the root of

343 Conidae, which corresponds with the first confident appearance of Conidae in the fossil record 
(Kohn 1990). We assigned 12 additional fossils (Table S2, Fig. S1 (Duda Jr. et al. 2001; Hendricks 2009, 2015, 2018)) to nodes throughout the phylogeny as minimum age constraints, which MCMCtree treats as soft bounds on the minimum age (Yang 2007). Further information on fossil placement on nodes can be found in the Supplementary. A recent paper showed that the number of species in Lautoconus may be overestimated (Abalde et al. 2017). To account for potential artificial inflation in the species richness of this clade, we artificially removed half the unique species in Lautoconus from our dataset and ran all dating analyses and downstream diversification analyses on this secondary dataset.

\section{Characterizing diversification patterns}

To visualize lineage accumulation patterns, we generated a log-lineage through time plot using the R package APE (Paradis et al. 2018). We estimated diversification rates and identified rate shifts using BAMM (Bayesian Analysis of Macroevolutionary Mixtures) (Rabosky 2014), which uses reversible jump Markov chain Mone Carlo to explore potential lineage diversification models. To account for non-randomness in species sampling across Conidae genera, we applied generic-specific sampling fractions. Using the number of valid Conidae names on WoRMS as estimates of total species diversity in each genus (Worms Editorial Board 2017), we applied a sampling fraction of $32.1 \%$ to Profundiconus, $50 \%$ to Lilliconus, $100 \%$ to Californiconus, $16.7 \%$ to Pygmaeconus, $28 \%$ to Conasprella, and 33.7\% to Conus. We ran BAMM for 100000000 generations and assessed convergence by calculating ESS values. We analyzed and visualized results using the R package BAMMtools (Rabosky et al. 2014).

\section{Trait dependent diversification}


We tested for the impact of evolvability (measured as conotoxin gene diversity) on diversification patterns using two trait dependent diversification methods, focusing on the genus

Conus. We focused our hypothesis testing on Conus because conotoxin diversity is well-

characterized in this group (Phuong et al. 2016) and the sequence capture approach used in this

contrast to other genera in Conidae, such as Conasprella or Profundiconus, where low conotoxin

373 diversity values are likely the result of poor knowledge of the venom repertoire of these genera

374 (Fig. S2)

First, we used BiSSE (binary state speciation and extinction, (Maddison et al. 2007))

376 implemeneted in the R package diversitree (FitzJohn 2012), which employs a maximum

377 likelihood approach to estimate the impact of a binary trait on speciation, extinction, and

378 transition rates between character states. We coded the conotoxin gene diversity data as 'low' or

379 'high' across several thresholds (i.e., 250, 300, 350, 400, 500, 550, or 600 estimated conotoxin

380 genes per species) and compared BiSSE models where speciation rates were allowed to vary or

381 remain equal between traits. We applied a sampling fraction of $33.7 \%$, taking the maximum

382 number of Conus species to be the number of valid names on WoRMS (World Register of

383 Marine Species, (Worms Editorial Board 2017)). We determined the best-fitting model using

384 Akaike Information Criterion (AIC). Second, we used FiSSE (Fast, intuitive State-dependent

385 Speciation-Extinction analysis), a non-parametric statistical test that assesses the effects of a

386 binary character on lineage diversification rates (Rabosky \& Goldberg 2017). We followed the

387 same coding strategy as in the BiSSE analyses to convert conotoxin gene diversity counts to

388 binary character states. Finally, we used STRAPP (Structured Rate Permutations on Phylogenies,

389 (Rabosky \& Huang 2016) implemented in the R package BAMMtools (Rabosky et al. 2014). 

impact of larval type on diversification patterns using BiSSE and FiSSE.

STRAPP is a semi-parametric approach that tests for trait dependent diversification by across the tips of the phylogeny (Rabosky \& Huang 2016). We generated the empirical permutations of evolutionary rates across the tree. We performed a two-tailed test with the gene diversity. Both piscivory and molluscivory is known to have evolved from the ancestral vermivory where speciation rates were allowed to vary or remained equal among traits. We excluded comparing a test statistic with a null distribution generated by permutations of speciation rates correlation (method $=$ Spearman's rank correlation) between speciation rates and conotoxin gene diversity and compared this test statistic with the null distribution of correlations generated by alternative hypothesis that there is a correlation between speciation rates and total conotoxin

We also tested the impact of diet and larval dispersal strategy on diversification patterns. condition in cone snails (Duda Jr. et al. 2001) and these diet transitions may be associated with increased diversification rates due to access to new dietary niches. In addition, differing larval dispersal strategies including long-lived larval stages (planktotrophy) and short-lived and/or direct developing larvae (lecithotrophy) are hypothesized to impact long term diversification patterns (Jablonski 1986). We coded diet as either vermivory, molluscivory, and piscivory using natural history information from (Jiménez-Tenorio \& Tucker 2013). We tested the impact of speciation and extinction using MuSSE (multistate speciation and extinction, (FitzJohn 2012)) species that were documented to feed on multiple diet types from this analysis. For larval type, we used protoconch morphology from (Jiménez-Tenorio \& Tucker 2013) to infer larval dispersal strategy, where multispiral protoconchs were indicative of planktotrophic larvae. We tested the 


\section{Results}

\section{Targeted sequencing data}

We sequenced an average of 9,548,342 reads (range: 1,693,918 - 29,888,444) across the

417363 samples (Table S1). After redefining exon/intron boundaries in the phylogenetic marker

418 reference, we ultimately targeted 2210 loci. On average, we recovered 1388 of these loci per

419 sample (range: $30-1849$, Table $\mathrm{S} 1$ ) at an average coverage of $12.39 \mathrm{X}$ (range: $3.08 \mathrm{X}-27.87 \mathrm{X}$,

420 Table S1). For the conotoxin dataset, each sequence we re-assembled contained a single

421 conotoxin exon with any associated noncoding regions (referred to here as 'conotoxin

422 fragments'). We recovered on average 3416 conotoxin fragments per sample (range: $74-11535$

423 fragments, Table $\mathrm{S} 1$ ) at an average coverage of 32.3X (range: 5.06X - 65.77X, Table S1). When

424 mapped to a reference containing both the phylogenetic markers and conotoxin genes, the \%

425 reads mapped to our targets was on average $14.86 \%$ (range: $0.7 \%-38.07 \%$, Table S1) and the

426 average level of duplication was $47.47 \%$ (range: $22.89 \%-89.06 \%$, Table S1).

We found that genus had an impact on \% mapped and \% duplication, where non-Conus

428 genera had lower \% mapping and lower \% duplication (Fig. S2). These differences likely

429

occurred because conotoxin fragments were not easily recovered in these genera (ANOVA, $\mathrm{p}<$

430 0.0001, Fig. S2). Genus did not have an impact on coverage or the number of phylogenetic

431 markers recovered (ANOVA, $p>0.05$, Fig. S2). We found that tissue quality, measured by the

432 maximum fragment length visualized via gel electrophoresis, had a significant impact on the

433 capture efficiency metrics (ANOVA, $p<0.0001$, Fig. S3). DNA samples with strong genomic

434 bands at the top of the gel tended to have higher \% mapping, less \% duplication, higher

435 coverage, and a greater number of targets recovered (Fig. S3). 
Our final conotoxin sequence dataset consists of exon fragments and we do not have

437 information on exon coherence (which exons pair together on the same gene). We were unable to

assemble full conotoxin genes because conotoxin introns are long ( $>1$ kilobases, (Wu et al.

$4392013)$ ) and exceed the average insert size of our sequencing experiment ( $350 \mathrm{bp})$. We recovered

440 fragments from all 58 gene superfamilies we targeted and obtained 159,670 sequences

441 containing some or all of the mature toxin region (Table S3). Total conotoxin gene diversity per

442 species (estimated by summing across all signal region exon fragments and sequences containing

443 the entire coding region) ranged from 5 to 1280 copies in Conus, 31 to 88 copies in

444 Profundiconus, and 7 to 164 in Conasprella (Table S1). Total conotoxin diversity was 311

445 copies for Californiconus californicus, 12 copies for Pygmaeconus tralli, and 30 copies for the

446 outgroup taxon, Bathyoma sp (Table S1). When compared to samples in (Phuong \& Mahardika

447 2017), the average change (increase or decrease) in total conotoxin gene diversity was $~ 90$ gene

448 copies (Table S4). If samples performed poorly in the number of phylogenetic markers

449 recovered, conotoxin gene diversity estimates tended to be lower in this study than in (Phuong \&

450 Mahardika 2017) and vice versa (Fig. S4). The average absolute change in the number of

451 fragments recovered per gene superfamily by region was 3.7 for sequences containing the signal

452 region, 12.2 for the prepro region, 9.6 for the mature region, 48.9 for the post region, and 3.4 for

453 sequences containing the entire coding region (Table S5, Fig. S5). We note several key outliers:

454 the average absolute change in the number of fragments was 104.3 for the $\mathrm{T}$ gene superfamily

455 containing the prepro region, 210.4 for the $\mathrm{O} 1$ gene superfamily prepro region, 57.4 for the $\mathrm{O} 1$

456 gene superfamily mature region, 219.9 for the $\mathrm{O} 2$ gene superfamily mature region, and 1417 for

457 the T gene superfamily post region (Table S5, Fig. S5). 
Phylogeny

The amount of missing data from the alignments was $15.4 \%$ when a minimum of $80 \%$ of the taxa were present in each locus, $26.8 \%$ when $50 \%$ of the taxa were present, and $38.6 \%$ when $20 \%$ of the taxa were present. The number of loci retained in the alignment was $387(107,011$ bp) when a minimum of $80 \%$ of the taxa were present in each locus, $976(237,027 \mathrm{bp})$ when $50 \%$ of the taxa were present, and 1476 loci $(336,557 \mathrm{bp})$ when $20 \%$ of the loci were present. Across all methods and datasets, we recovered phylogenies with a moderate level of resolution (average number of nodes resolved $=71.1 \%$, range $=61.4-79.2 \%$, Table S6). In general, as increased amounts of sequence data was given to the phylogenetic programs, more nodes became resolved (Table S6). While we recovered all 6 genera within Conidae with high confidence, relationships among subgenera were less supported (bootstrap and PP = 100\%, Fig. 1, Fig. S6, S7, S8).

\section{Divergence time estimation}

We found evidence for three major branching events during the Eocene: (1) a branching event leading to Profundiconus (56.5 mya, CI = 46.3 - 65.3 mya, Fig. 1, S9), (2) a branching event leading to Conus (54.7 mya, CI = 42.5 - 63.6 mya, Fig. 1, S9), and (3) a branching event separating Conasprella and Californiconus, Lilliconus, and Pygmaeconus (46.0 mya, CI = 36.5 53.2 mya, Fig. 1, S9). The branching event leading to Californiconus occurred during the Oligocene (26.1 mya, CI = 13.8 - 36.5 mya, Fig. 1, S9) and the split between Lilliconus and Pygmaeconus occurred during the Miocene (17.8 mya, CI =9.25 - 25.1 mya, Fig. 1, S9). 
We found that most branching events within each genus began to occur in the Miocene and continued until the present (Fig. 1). When analyzing the entire dataset, we found support for diversification rate heterogeneity, where BAMM identified at least one rate shift across Conidae

484 (Fig. 1, S10). Across the 95\% credible set of distinct shift configurations, BAMM detected an 485 increase in diversification rates on the branch leading to Lautoconus, a clade consisting mainly

486 of species endemic to the Cape Verde islands (Fig. 1, S10). However, when examining an

487 artificially reduced dataset consisting of half the species within Lautoconus, we detect no rate 488 shift or a decrease in diversification rates leading to the Conus clade (Fig. S11).

\section{Trait dependent diversification}

Across all thresholds for the BiSSE analysis, we found that diversification rates were not

492 influenced by conotoxin gene diversity. In all cases, the null model was either preferred (delta

493 AIC > 2, Table S7) or was indistinguishable from a model where speciation and extinction were 494 allowed to vary (delta AIC < 2, Table S7). Both the FiSSE and STRAPP analyses revealed that 495 speciation rates were not correlated with conotoxin gene diversity $(\mathrm{p}>0.05)$. These results were 496 consistent across both the full dataset and the reduced dataset.

We found that diversification rates were not dependent on diet when analyzing the full

498 dataset (Table S8). However, in the reduced dataset, we found a signal for diet-dependent 499 speciation rates (delta AIC > 2, Table S8). We found that species with mollusk-feeding diets had 500 the fastest speciation rates $(0.33)$, followed by piscivory $(0.24)$, and vermivory (0.16). For the 501 larval dispersal trait, we found support for trait-dependent speciation rates in the full dataset 502 (delta AIC > 2, Table S9), where species with short-lived larvae had higher speciation rates $(0.27$ 
503 vs. 0.16). However, this result was not significant when examining the reduced dataset (Table

504 S9).

505

506

507 Discussion

508

509

Capture results

Our targeted sequencing experiment underperformed initial testing of this sequencing method on cone snails (Phuong \& Mahardika 2017). Although tissue quality impacted capture

512 metrics (Fig. S3), the \% of reads mapping to our targets for even our best samples was $\sim 30 \%$

513 lower than expected (Phuong \& Mahardika 2017). While it is difficult to determine the exact

514 cause of this depression in our capture statistics, we hypothesized that changes made in the bait

515 design between this study and (Phuong \& Mahardika 2017) may have led to poorer capture

516 results. For example, we recovered an overabundance of conotoxin sequences containing the post

517 region from the $\mathrm{T}$ gene superfamily that has no clear co-variation pattern with phylogenetic

518 relatedness (Fig. S12), which likely indicates a large amount of non-specific binding due to

519 conotoxin misclassification. In the future, we suggest re-designing the baits to only include

520 sequences from only the most critical regions (signal region and mature region) to avoid non-

521 specific binding. Although overall capture efficiency statistics were low, the absolute change in

522 conotoxin diversity estimates per gene superfamily was generally minor (Table S5). Therefore,

523 we do not believe that total conotoxin diversity metrics were severely biased by the sequencing

524 method. 
Phylogenetic relationships

Below, we discuss the results of our phylogenetic analyses, how the phylogenetic relationships compare to past work, and their implications for Conidae taxonomy. Unless otherwise noted, the results we highlight below have at least $90 \%$ bootstrap support in the RAxML analyses and 90\% posterior probabilities from the ASTRAL-II analyses (Figure S7, S8).

When present results on subgeneric relationships starting from the top of the tree shown in Figure S6.

We recovered all six major deep lineages representing genera in Conidae that were previously described in recent molecular phylogenetic studies using mtDNA (Puillandre et al. 2014a; Uribe et al. 2017), Fig. 1, S6, S7, S8). Specifically, we find strong support for Profundiconus, Californiconus, Lilliconus, Pygmaeconus, Conasprella, and Conus, as separate and distinct lineages. We also confirm the branching order of these six genera that were recently described using mtDNA genomes (Uribe et al. 2017), with Profundiconus being sister to all other genera, Pygmaeconus + Lilliconus sister to Californiconus, Californiconus + Lilliconus + Pygmaeconus sister to Conasprella, and these four genera sister to Conus.

Based on the molecular phylogeny from three mtDNA genes, monophyletic groupings of species from Conasprella were classified into several subgenera (Puillandre et al. 2014a; b). We note several differences between past results and our study in the relationships among these genera and their monophyly:

(1) Ximeniconus is sister to all other Conasprella in some trees, or we reconstructed a polytomy at the base of Conasprella, which contrasts with Conasprella (Kohniconus) arcuata recovered at the base of Conasprella in previous work (Puillandre et al. 2014a). 
(2) Kohniconus is polyphyletic. In (Puillandre et al. 2014a), only a single species from Kohniconus was included and we find evidence for the non-monophyly of Kohniconus when we included the additional species, C. centurio. Given these results, we propose that $C$. emarginatus, $C$. delssertii, and $C$. centurio be placed in the subgenus Kohniconus and $C$. arcuata placed in a new subgenus.

(3) Endemoconus is paraphyletic. When including an additional species (C. somalica) not sequenced in (Puillandre et al. 2014a), we find that Endemoconus is not monophyletic. Based on these results, C. somalica should be transferred to Conasprella.

Within Conus, our results largely confirm previous findings that $C$. distans is sister to all other Conus species and the relationships among subgenera remain tenuous and difficult to resolve (Puillandre et al. 2014a). We note the following differences in subgenera relationships and classification between our results and past work:

(1) We found support the sister relationship between Turriconus and Stephanoconus, which has not been recovered in a previous study (Puillandre et al. 2014a).

(2) We found support for the monophyly of Pyruconus across our RAxML analyses, but not our ASTRAL-II analyses. The monophyly of Pyruconus was not supported in (Puillandre et al. 2014a).

(3) C. trigonus and C. lozeti were classified into the subgenus (Plicaustraconus) based on morphological characters (Jiménez-Tenorio \& Tucker 2013; Puillandre et al. 2014b). We found this subgenus to be polyphyletic when sequence data was obtained.

(4) Similar to (Puillandre et al. 2014a), we found that Textila + Afonsoconus is sister to Pionoconus. However, instead of the unsupported relationship of Asprella as sister to these three subgenera, we found support for Gastridium as the sister group. 
(5) We found support for the sister relationship between Asprella and Phasmoconus, which conflicts with the unsupported relationship shown (Puillandre et al. 2014a), where these subgenera branch in different parts of the phylogeny.

(6) We find support for the following successional branch order: Tesselliconus, Plicaustraconus, Eugeniconus, and Conus. We found that Conus is sister to Leptoconus, Darioconus, and Cylinder, but the relationships among these three subgenera remained unresolved. This conflicts with (Puillandre et al. 2014a) as Cylinder was paraphyletic, whereas in our results with increased sampling of Eugeniconus, Cylinder became monophyletic.

(7) We did not find strong support for the subgenus Calibanus, contrasting with previous work (Puillandre et al. 2014a). In our results, we found that $C$. thalassiarchus and $C$. furvus were not sister to each other, or their relationship resulted in an unresolved polytomy. Additional investigation into the subgeneric status of these two species.

(8) C. sanderi was classified into its own subgenus (Sandericonus) based on morphological characters (Jiménez-Tenorio \& Tucker 2013; Puillandre et al. 2014b). Here, when sequence data were obtained, we found it nested within Dauciconus. Therefore, we synonymize Sandericonus with Dauciconus because $C$. sanderi is the type species for Sandericonus.

(9) C. granulatus was classified into its own subgenus (Atlanticonus) based on morphological characters (Jiménez-Tenorio \& Tucker 2013; Puillandre et al. 2014b). Here, we found that it was nested within Dauciconus. No other species within this subgenus have been sequenced up until this point. Therefore, we synonymize Atlanticonus with Dauciconus because $C$. granulatus is the type species for Atlanticonus. 

marine snails. into the subgenus Elisaconus (Puillandre et al. 2014b). Our results do not support the monophyly of Elisaconus, as the sister relationship between $C$. moncuri and $C$. pergrandis was not supported in 5/6 trees. Additional data is required to classify $C$. moncuri and $C$. pergrandis into the appropriate subgenus.

(11) C. cocceus was placed into Floraconus based on morphological characters in (Puillandre et al. 2014b). With sequence data, we found that it was actually nested within Phasmoconus. Therefore, we transfer C. cocceus to the subgenus, Phasmoconus.

Classification within Conidae is known to be highly unstable (Jiménez-Tenorio \& Tucker 2013; Puillandre et al. 2014a; b; Puillandre \& Tenorio 2018). Although the phylogeny presented here improved understanding of subgeneric relationships and monophyly of subgenera, resolving relationships within Conidae still remains a significant challenge. Given the underperformance of our capture experiment (Table S1), it is unclear if the reason for the moderate power in resolving relationships is due to insufficient data/incomplete data or due to short internal branches during the origination of Conidae subgenera that are extremely difficult to resolve. Overall, our results suggest that both additional data and increased sampling of Conidae species are reasonable pursuits to continue attempting to resolve the phylogeny and classification of this family of

\section{Timing of diversification}

The timing of splits between major are largely congruent with past estimates from a study using mtDNA genomes (Uribe et al. 2017), Fig. 1, S9). However, our age estimates for the 
617 branching events between Californiconus, Lilliconus, and Pygmaeconus are much younger

618 (occurring across the Oligocene into the Miocene) than previous estimates (occurring across the

619 Eocene into the Oligocene, (Uribe et al. 2017), Fig. 1, S9). This discrepancy may have been

620 caused by differences in fossil calibration, as we included many more fossils in this study

621 compared to previous studies. The Conidae fossil record and analyses of several molecular

622 phylogenetic studies suggest a major radiation of Conus during the Miocene (Kohn 1990; Duda

623 Jr. et al. 2001; Uribe et al. 2017). While we noted that many branching events within Conus

624 ocurred during the Miocene into the present, we did not detect an increase in diversification on

625 the branch leading to the origin of Conus (Fig. 1, S10, S11). This is congruent with

626 diversification rates estimated from the fossil record (Kohn 1990), suggesting that the

627 accumulation of species during the Miocene may have been a function of an increased number of

628 lineages present rather than an increase in diversification rates. The number of species we

629 included in the subgenus Lautoconus had an impact on the BAMM diversification analyses. On

630 the full dataset, BAMM detected an increase in diversification rates leading to Lautoconus (Fig.

631 1, S10), a known and documented radiation of cone snails (Duda \& Rolán 2005; Cunha et al.

632 2005). However, when we remove half the species in response to recent work suggesting

633 taxonomic inflation in this subgenus (Abalde et al. 2017), we do not detect the same shift.

634 Rather, there is partial support for no shift across Conidae, or a slight decrease in diversification rates leading to Conus (Fig. S11). These results suggest that the original diversification analyses

636 and identified radiation of Lauotoconus may have been due to taxonomic inaccuracies biasing

637 the diversification analyses results, rather than a true radiation. What is even more striking about

638 these results is that we found minimal diversification rate heterogeneity across Conidae, despite the expansive species richness across this group. It is unclear whether this signal is real, or due to 
640 other technical artifacts. For sample, although we included over 300 species in this study, this

641 only represents $\sim 30 \%$ of the total diversity in this group and may have hindered our ability to

642 effectively estimate diversification rates. Similarly, new Conidae species are continually

643 described, with over 100 species described over the last few years (Worms Editorial Board

644 2017). Therefore, our inability to estimate the number of living taxa may have weakened our

645 ability to test the impact of diversification on this group.

646

647

Speciation rates and conotoxin gene diversity

Contrary to macroevolutionary expectations, we were unable to detect any relationship

655 Fig. 1, S10, S11). As discussed previously, taxonomic instability in this group may have

656 hindered our efforts to estimate past historical diversification patterns. However, we did find

657 some signal for the impact of diet and larval dispersal strategy on diversification rates when

658 using the BiSSe and MuSSE methods (Table S8, S9). Further work is needed to be fully

659 confident in this signal given high false positive rates in these methods (Abosky 2017; Rabosky

$660 \&$ Goldberg 2017) and given that our results depended on which dataset was used.

What is remarkable about these results is the lack of any signal on the impact of venom

662 gene diversity on diversification rates in cone snails, even as we found some signal for trait- 
663 dependent diversification in other Conidae characters. If this lack of signal is real, several

664 biological factors may explain this decoupling between conotoxin gene diversity and speciation

665 rates. A critical assumption in Conus biology is that ecological diversification driven by diet

666 specialization is a major factor governing diversification dynamics in cone snails (Duda \&

667 Palumbi 1999; Duda Jr. et al. 2001). Past studies have shown that cone snail venom repertoires

668 track their dietary breadth, providing a link between diet and venom evolution (Phuong et al.

669 2016; Phuong \& Mahardika 2017). However, it is unclear whether or not the relationship

670 between diet and venom evolution leads to ecological speciation due to divergence in prey

671 preference. Ecological speciation is often difficult to detect in marine ecosystems and long-term

672 diversification patterns may be better explained by traits that limit dispersal and promote

673 isolation (Bowen et al.). Another possibility is that conotoxin phenotypic divergence may not be

674 the rate-limiting factor in prey specialization and divergence (Duda Jr. et al. 2001). Conotoxin

675 genes are under continuous positive selection and gene duplication that allow venom components

676 to change rapidly in response to the environment (Duda \& Palumbi 1999; Duda Jr. et al. 2001;

677 Chang \& Duda 2012; Phuong \& Mahardika 2017). This persistent evolutionary change in the

678 venom cocktail suggests that perhaps venom evolution is not necessarily the factor limiting

679 dietary shifts among species and ultimately, speciation among taxa. Ecological opportunity is

680 hypothesized as a necessary component for diversification (Losos 2010) and may be a more

681 critical factor limiting Conidae diversification. Indeed, evidence from the fossil record and past

682 Conidae molecular phylogenetic studies indicate a concentration of lineage formation during the

683 Miocene (Kohn 1990; Duda Jr. et al. 2001; Uribe et al. 2017), a period that is coincident with the

684 formation of coral reefs in the Indo-Australian Archipelago (Cowman \& Bellwood 2011). Our

685 results also show a concentration of branching events during this period as well, though we do 
not detect a shift in diversification rates (Fig. 1, S10, S11). Overall, our results point to increased taxonomic sampling and a holistic approach to investigating factors shaping diversification in Conidae for future work.

Venom evolution is assumed to be a key innovation that led to the evolutionary success of venomous animal lineages (Pyron \& Burbrink 2011; Sunagar et al. 2016) and a large body of work is devoted towards understanding how venom evolves and responds to the environment over time (Kordis \& Gubensek 2000; Wong \& Belov 2012; Casewell et al. 2013). However, the impact of venom evolution on higher-level diversification patterns is rarely tested. Here, we examined the effect of variation in the adaptive capacity of venom across Conidae species and found it had no influence on macroevolutionary diversification patterns. Although we do not detect a strong signal of conotoxin gene diversity shaping speciation rates in Conidae, it does not refute the importance of venom evolution in adaptation and prey specialization as venom may be necessary, but not sufficient, to promote speciation (Duda et al. 2009; Safavi-Hemami et al. 2015; Chang \& Duda 2016; Phuong et al. 2016; Phuong \& Mahardika 2018). Future work in other venomous animal systems may shed light on whether or not the ability to adapt to different prey through venom evolution translates to the long-term evolutionary success of taxa.

\section{Data availability}

Raw read data will be made available at the National Center for Biotechnology Information Sequence Read Archive. Bait sequences, conotoxin sequences, scripts, and final datasets used for analyses will be uploaded onto Dryad following publication.

\section{Acknowledgements}


709

710

711

712

713

714

715

716

717

718

719

720

721

722

723

724

725

726

727

728

729

730

731

A majority of the sampling material in this paper originates from numerous shore-based expeditions and deep sea cruises, conducted respectively by MNHN and Pro-Natura International (PNI) as part of the Our Planet Reviewed programme (SANTO 2006, ATIMO VATAE, MAINBAZA, INHACA 2011, GUYANE 2014, PAPUA NIUGINI, KAVIENG 2014), by MNHN and AAMP (Pakaihi i Te Moana), and/or by MNHN and Institut de Recherche pour le Développement (IRD) as part of the Tropical Deep-Sea Benthos programme (AURORA 2007, BIOPAPUA, EBISCO, EXBODI, MADEEP, MIRIKY, TAIWAN 2013, NANHAI 2014, BIOPAPUA, SALOMONBOA 3, CONCALIS, EXBODI, SALOMON BOA3, KARUBENTHOS 2015, NORFOLK 2, TERRASSES). Scientific partners included the University of Papua New Guinea (UPNG); National Fisheries College, Kavieng; Institut d'Halieutique et Sciences Marines (IH.SM), Université de Tuléar, Madagascar; Universidade Eduardo Mondlane, Maputo; the Madagascar bureau of the Wildlife Conservation Society (WCS); and Instituto Español de Oceanografia (IOE). Funders and sponsors included the Total Foundation, Prince Albert II of Monaco Foundation, Stavros Niarchos Foundation, Richard Lounsbery Foundation, Vinci Entrepose Contracting, Fondation EDF, European Regional Development Fund (ERDF), the Philippines Bureau of Fisheries and Aquatic Research (BFAR), the French Ministry of Foreign Affairs, Fonds Pacifique and the Government of New Caledonia. Additional field work included PANGLAO 2004 and PANGLAO 2005 (joint projects of MNHN and University of San Carlos, Cebu City, and the Philippines Bureau of Fisheries and Aquatic Research); KARUBENTHOS 2012 (a joint project of MNHN with Parc National de la Guadeloupe and Université des Antilles); sampling in Western Australia arranged by Hugh Morrison, with support of the Western Australian Museum. The Taiwan and South China Sea cruises were supported by bilateral cooperation research funding from the Taiwan Ministry of 
Science and Technology (MOST 102-2923-B-002-001-MY3, PI Wei-Jen Chen) and the French National Research Agency (ANR 12-ISV7-0005-01, PI Sarah Samadi). All expeditions operated under the regulations then in force in the countries in question and satisfy the conditions set by the Nagoya Protocol for access to genetic resources. We thank the Indonesian Ministry of State for Research and Technology (RISTEK) for providing permission to MAP and GNM to conduct fieldwork Bali in 2014 (permit number 277/SIP/FRP/SM/VIII/ 2013) and providing permission to PWHV TvR RMM and REP to conduct fieldwork across Indonesia in 2016 (permit number XXXXXXXXXX). We thank DST Hariyanto, MBAP Putra, MKAA Putra, and the staff at the Indonesian Biodiversity Research Center in Denpasar, Bali for assistance during the 2014 field season; the staff at the Museum Zoologicum Bogoriense for assistance during the 2016 field season; F Criscione, F Köhler, A Moussalli, A Hogget, and L Vail for logistical assistance for fieldwork at the Lizard Island Research Station in Australia; M Reed, A Hallan, and J

Waterhouse for access to specimens at the Australian Museum in Sydney, Australia; J Finn, M Mackenzie, and M Winterhoff for access to specimens at the Museum Victoria in Melbourne, Australia; G Pauley and AM Bemis for access to specimens at the Florida Museum of Natural History at the University of Florida; TF Duda Jr. and T Lee for access to specimens at the University of Michigan Museum of Zoology; L Kirkendale and C Whisson for access to specimens at the Western Australian Museum in Perth, Australia; WF Gilly for access to the $C$. californicus specimen; H Safavi-Hemami and Q Li for access to 10 additional Conidae transcriptomes; K Bi for advice on bait design; A Devault and MYcroarray for great service and technical support for bait synthesis; L Smith and the Evolutionary Genetics Lab at UC Berkeley for laboratory support; J Chang, MCW Lim and EM McCartney-Melstad for thoughtful advice and discussions throughout the entire process; E Monnier for collating information on Conidae 
755 larval dispersal strategies and MJ Tenorio for verifying Conidae diets and identifications. This work used the Extreme Science and Engineering Discovery Environment (XSEDE), which is supported by National Science Foundation grant number ACI-1053575. This work was supported by two Grants-in-Aid of research from Sigma Xi, a Grants-in-Aid of Research from the Society for Integrative and Comparative Biology, a research grant from the Society of Systematic Biologists, a Student Research Award from the American Society of Naturalists, a National Science Foundation Graduate Research Opportunities Worldwide to Australia, the Lerner Gray Fund for Marine Research from the American Museum of Natural History, research grants from the Department of Ecology and Evolutionary Biology at UCLA, the Melbourne R.

765 from the Conchologists of America, a Student Research Award from Unitas Malacologicas, a

767 from the American Philosophical Society, a Young Explorer's Grant from the National

768 Geographic Society, a Travel Award from the UCLA Graduate Division, a Research Grant from

769 the American Institute for Indonesian Studies and the Council of American Overseas Research

770 Centers, a small award from the B Shaffer Lab, a National Science Foundation Graduate

771 Research Fellowship, an Edwin W. Pauley fellowship, a Fulbright Fellowship to Indonesia, and a

772 Chateaubriand fellowship awarded to MAP. This work was supported by the Service de

773 Systématique Moléculaire (UMS 2700 CNRS-MNHN) and the CONOTAX project funded by

774 the French National Research Agency (grant number ANR-13-JSV7-0013-01). This work used

775 the Vincent J. Coates Genomics Sequencing Laboratory at UC Berkeley, supported by NIH S10

776 OD018174 Instrumentation Grant. The C. californicus specimen was collected under a

777 California Department of Fish and Wildlife collecting permit granted to WF Gilly (SC-6426). 
779 Figure 1. Time calibrated maximum likelihood phylogeny of the cone snails. Phylogeny was estimated in RAxML using a concatenated alignment of loci and was calibrated using 13 fossils placed at nodes throughout the tree. Only loci with at least $20 \%$ of the taxa present were included in the alignment. Colors across the phylogeny show instantaneous diversification rates and are averaged across all rate models sampled from a BAMM analysis. Warmer colors indicate higher speciation rates. Log-lineage through time plot is shown below the phylogeny. First four columns shown next to tip represent the following from left to right: presence of vermivory (blue), presence of molluscivory (blue), presence of piscivory (blue), larval type (planktotrophy: tips depicting variation conotoxin gene diversity across the phylogeny. If bar is not shown, data is not available or were excluded from downstream diversification analyses. Histogram on the bottom right shows variation in conotoxin gene diversity. Abbreviations: Plio. $=$ Pliocene; Pleis. $=$ Pleistocene.

Table S1. Sample information and capture efficiency metrics. We first list the species name listed in all phylogenetic analyses ("species") and the accepted taxonomic classification in the

794 WoRMS database at the genus, subgenus, and species level ("WoRMS genus", "WoRMS subgenus", "WoRMS species"). We then list the specimen ID ("ID"), the collection source 796 ("Collection"), the year the sample was collected ("Year Collected"), how the sample was 797 preserved ("Preservation type"), and the country the sample originated from ("Country"). We 798 then list "Gelsize", or the largest fragment size visualized via gel electrophoresis as a way to 799 measure tissue quality. Values can be either "g" for genomic band, or 1500, 1000, or 500 for bands beginning at $1500 \mathrm{bp}, 1000 \mathrm{bp}$, or $500 \mathrm{bp}$. We list the data collection method ("Data 
801 collection method"), the number of reads sequenced (“\# of reads sequenced") and several capture

802 efficiency metrics. Finally, we list the total estimated number of conotoxin genes per species

803 (“Conotoxin gene diversity”).

804

805

Table S2. Information on fossils used for calibration. We list the fossil taxon ("Fossil Species"),

806 the genus (“Clade assignment"), extant species related to the fossil (“Compared with”), the

807 formation ("Formation"), the age of the fossil ("Age"), and its citation ("Reference").

808

809 Table S3. Table showing the number of conotoxin sequences recovered per species for each

810 gene superfamily. Within each gene superfamily, conotoxin sequences were categorized based

811 on whether they contained the entire coding region or mostly the signal, prepro, mature, or post

812 regions.

813

814 Table S4. Comparison of conotoxin gene diversity estimates between this study and (Phuong \&

815 Mahardika 2017). These represent comparisons between technical replicates (capture experiment

816 was performed on the same libraries in both studies).

817

818 Table S5. Comparison of conotoxin gene diversity estimates between this and (Phuong \&

819 Mahardika 2017), broken down by gene superfamily. Within each gene superfamily, conotoxin

820 sequences were categorized based on whether they contained the entire coding region or mostly

821 the signal, prepro, mature, or post regions. These represent comparisons between technical

822 replicates (capture experiment was performed on the same libraries in both studies). 
824 Table S6. Number of nodes resolved depending on the amount of missing data and the tree

825

826

827

828

829

830

831

832

833

834

835

836

837

838

839

840

841

842 843 (speciation rates across trait states were equal).

844

845

846 were not allowed to vary by trait. trait states were equal).

Table S7. Venom gene diversity BiSSE AIC results. "Dataset" represents whether the full dataset was used or the reduced dataset. "Threshold" represents the conotoxin gene diversity value used to decide between "high" and "low" conotoxin diversity. Values above the threshold value were categorized as "high" and values below were categorized as "low". "AIC - variable rates" shows AIC values for a model where speciation and extinction rates were allowed to vary depending on a trait. "AIC - equal rates" represents AIC values for the null model, where rates

Table S8. Diet BiSSE AIC results. Model values were generated under a variable rates model (where speciation was allowed to vary) or under an equal rates model (speciation rates across

Table S9. Larval dispersal type BiSSE AIC results. Model values were generated under a variable rates model (where speciation was allowed to vary) or under an equal rates model

$$
\text { Figure S1. Node placement of fossils. Numbers correspond to node placement justification in }
$$
the supplementary information on node assignment. Tree was generated from a RAxML analysis 
847 of a concatenated alignment where loci were kept if at least $20 \%$ of species was present in the

848 locus. Best tree is shown and erroneous and intraspecific tips were pruned.

849

850 Figure S2. Boxplots showing impact of phylogeny (categorized by Conidae genus) on capture

851 efficiency metrics. Graph title shows resultant P value from ANOVA analyses.

852

853 Figure S3. Boxplots showing impact of tissue quality (estimated by maximum DNA fragment

854 lengths assessed via gel electrophoresis) on capture efficiency metrics. Categories are either "g"

855 for genomic band, or 1500, 1000, or 500 for bands beginning at 1500bp, 1000bp, or 500bp.

856 Graph title shows resultant P value from ANOVA analyses.

857

858

Figure S4. Scatterplot showing relationship between the number of phylogenetic markers

859 recovered and the change in total conotoxin gene diversity between this study and (Phuong \&

860 Mahardika 2017). Results showed a positive relationship between the two parameters, suggesting

861 that if a sample performed poorly in the capture experiment, it performed poorly in recovering

862 data across all loci (phylogenetic loci or conotoxin loci).

863

864 Figure S5. Histograms showing absolute change in conotoxin sequence diversity per gene

865 superfamily between this study and (Phuong \& Mahardika 2017). Graphs are partitioned by

866 conotoxin functional region, where sequences were categorized based on whether they contained

867 the entire coding region or mostly the signal, prepro, mature, or post regions. On average,

868 estimates of conotoxin diversity per gene superfamily varied slightly. 
870 Figure S6. Maximum likelihood phylogeny inferred using RAxML, where $20 \%$ of the taxa

871 needed to be present within a locus to be included in the final concatenated alignment. The six

872 major genera are colored and subgenera re noted for Conasprella and Conus.

873

874 Figure S7. Phylogenies inferred through the coalescent-based method, ASTRAL-II. Individual

875 loci were inferred under default paramenters in RAxML. Nodes are collapsed when posterior

876 probailities are $<90 \%$. Trees are colored and labeled by genus. We varied the level of missing

877 data for each ASTRAL run, where we only retained loci if (a) $80 \%$ of taxa had sequences, (b)

$87850 \%$ had sequences, and (c) $20 \%$ of taxa had sequences.

879

880

Figure S8. Maximum likelihood phylogenies generated using a concatenated alignment. Nodes are collapsed when boostrap support values are $<90 \%$. Trees are colored and labeled by genus.

882 We varied the level of missing data for each RAxML run, where we only retained loci for the nal concatenated alignment if (a) $80 \%$ of taxa had sequences, (b) $50 \%$ had sequences, and (c) $20 \%$ of taxa had sequences.

Figure S9. Maximum likelihood phylogeny dated with 13 fossil node calibrations in MCMCtree.

$88795 \%$ confidence intervals shown at nodes. The final concatenated alignment consisted of loci 888 where $20 \%$ of the taxa needed to be present within the locus to be included.

Figure S10. 95\% credible set of distinct shift configurations from BAMM for the full dataset.

891 Each graph is labeled by the posterior probability of each shift configuration. Warmer, red colors 
892

893

894

895

896

897

898

899

900

901

902

903

904

905

906

907

908

909

910

911

912

913

914

represent faster speciation rates than cooler, blue colors. We note that in all shift configurations, there is a shift in diversification rates in the clade leading to Lautoconus.

Figure S11. 95\% credible set of distinct shift configurations from BAMM for the reduced dataset. Each graph is labeled by the posterior probability of each shift configuration. Warmer, red colors represent faster speciation rates than cooler, blue colors. We note that in all shift configurations, there is a shift in diversification rates in the clade leading to Lautoconus.

Figure S12. Diversity estimates for the A gene superfamily signal region and the T gene superfamily post region. Estimates are plotted next to the RAxML phylogeny where $20 \%$ of taxa had sequences in each locus.

Abalde S, Tenorio MJ, Afonso CML et al. (2017) Phylogenetic relationships of cone snails endemic to Cabo Verde based on mitochondrial genomes. , 1-19.

Abosky DALR (2017) Model Inadequacy and Mistaken Inferences of Trait-Dependent Speciation. , 64, 340-355.

Ashkenazy H, Penn O, Doron-faigenboim A et al. (2012) FastML : a web server for probabilistic reconstruction of ancestral sequences. , 40, 580-584.

Benson G (1999) Tandem Repeats Finder: a program to analyse DNA sequences. Nucleic Acids Res., 27, 573-578.

Bi K, Vanderpool D, Singhal S et al. (2012) Transcriptome-based exon capture enables highly cost-effective comparative genomic data collection at moderate evolutionary scales. $B M C$ Genomics, 13, 403. 
915 Bowen BW, Rocha LA, Toonen RJ, Karl SA The origins of tropical marine biodiversity.

916 Casewell NR, Wüster W, Vonk FJ, Harrison RA, Fry BG (2013) Complex cocktails: the evolutionary novelty of venoms. Trends in ecology \& evolution, 28, 219-29.

918 Chang D, Duda TF (2012) Extensive and continuous duplication facilitates rapid evolution and diversification of gene families. Molecular biology and evolution, 29, 2019-29.

Chang D, Duda TF (2016) Age-related association of venom gene expression and diet of predatory gastropods. BMC Evolutionary Biology, 16, 27.

Cowman PF, Bellwood DR (2011) Coral reefs as drivers of cladogenesis: expanding coral reefs, cryptic extinction events, and the development of biodiversity hotspots. Journal of evolutionary biology, 24, 2543-2562.

Crow KD, Wagner GP (2006) What is the role of genome duplication in the evolution of complexity and diversity? Molecular biology and evolution, 23, 887-92.

Cunha RL, Castilho R, Rüber L, Zardoya R (2005) Patterns of cladogenesis in the venomous marine gastropod genus Conus from the Cape Verde islands. Systematic biology, 54, 63450.

Duda TF, Chang D, Lewis BD, Lee T (2009) Geographic variation in venom allelic composition and diets of the widespread predatory marine gastropod Conus ebraeus. PloS one, 4, e6245.

Duda Jr. TF, Kohn AJ, Palumbi SR (2001) Origins of diverse feeding ecologies within Conus, a genus of venomous marine gastropods. Biological Journal of the Linnean Society, 73, 391409.

Duda TF, Palumbi SR (1999) Molecular genetics of ecological diversification: duplication and rapid evolution of toxin genes of the venomous gastropod Conus. Proceedings of the National Academy of Sciences of the United States of America, 96, 6820-3. 
Duda TF, Palumbi SR (2000) Evolutionary diversification of multigene families: allelic selection of toxins in predatory cone snails. Molecular biology and evolution, 17, 1286-93.

Duda TF, Rolán E (2005) Explosive radiation of Cape Verde Conus, a marine species flock. Molecular ecology, 14, 267-72.

FitzJohn RG (2012) Diversitree : comparative phylogenetic analyses of diversification in R. Methods in Ecology and Evolution, 3, 1084-1092.

Folmer O, Black M, Hoeh W, Lutz R, Vrijenhoek R (1994) DNA primers for amplification of mitochondrial cytochrome c oxidase subunit I from diverse metazoan invertebrates. Molecular marine biology and biotechnology, 3, 294-9.

Grabherr MG, Haas BJ, Yassour M et al. (2011) Full-length transcriptome assembly from RNASeq data without a reference genome. Nature biotechnology, 29, 644-52.

Hendricks JR (2009) The Genus Conus (Mollusca:Neogastropoda) in the Plio-Pleistocene of the Southeastern United States. Paleontological Research Institution, Ithaca, NY.

Hendricks JR (2015) Glowing Seashells : Diversity of Fossilized Coloration Patterns on Coral Reef-Associated Cone Snail (Gastropoda : Conidae) Shells from the Neogene of the Dominican Republic. Plos One, 10, 1-59.

Hendricks JR (2018) Diversity and preserved shell coloration patterns of Miocene Conidae (Neogastropoda) from an exposure of the Gatun Formation, Colón Province, Panama. Journal of Paleontology, In press.

Hoekstra HE, Coyne JA (2007) The Locus of Evolution: Evo Devo and the Genetics of Adaptation. Evolution, 61, 995-1016.

Huang X, Madan A (1999) CAP3: A DNA sequence assembly program. Genome Research, 9, $868-877$. 
Jablonski D (1986) Larval ecology and macroevolution in marine invertebrates. Bulletin of Marie Science, 39, 565-587.

Jiménez-Tenorio M, Tucker JK (2013) Illustrated catalog of the Living Cone snails. MdM Publishing, Wellington, FL.

Jones AG, Arnold SJ, Bürger R (2007) The mutation matrix and the evolution of evolvability. Evolution, 61, 727-45.

Kaas Q, Westermann J-C, Craik DJ (2010) Conopeptide characterization and classifications: an analysis using ConoServer. Toxicon, 55, 1491-509.

Kaas Q, Yu R, Jin A-H, Dutertre S, Craik DJ (2012) ConoServer: updated content, knowledge, and discovery tools in the conopeptide database. Nucleic acids research, 40, D325-30.

Katoh K, Kuma K-I, Toh H, Miyata T (2005) MAFFT version 5: Improvement in accuracy of multiple sequence alignment. Nucleic Acids Research, 33, 511-518.

Kohn AJ (1990) Tempo and mode of evolution in conidae. Malacologia, 32, 55-67.

Kohn AJ (2001) Maximal species richness in Conus: diversity, diet and habitat on reefs of northeast Papua New Guinea. Coral Reefs, 20, 25-38.

Kordis D, Gubensek F (2000) Adaptive evolution of animal toxin multigene families. Gene, 261, $43-52$.

Langmead B, Salzberg SL (2012) Fast gapped-read alignment with Bowtie 2. Nature methods, 9, $357-9$.

Lavergne V, Harliwong I, Jones A et al. (2015) Optimized deep-targeted proteotranscriptomic profiling reveals unexplored Conus toxin diversity and novel cysteine frameworks. Proceedings of the National Academy of Sciences, 112.

Li W, Godzik A (2006) Cd-hit: a fast program for clustering and comparing large sets of protein 
or nucleotide sequences. Bioinformatics, 22, 1658-9.

985

986

987

988

989

990

Li H, Handsaker B, Wysoker A et al. (2009) The Sequence Alignment/Map format and SAMtools. Bioinformatics, 25, 2078-9.

Losos JB (2010) Adaptive radiation, ecological opportunity, and evolutionary determinism. The American naturalist, 175, 623-639.

Maddison WP, Midford PE, Otto SP (2007) Estimating a binary character's effect on speciation and extinction. Systematic biology, 56, 701-10.

Magoč T, Salzberg SL (2011) FLASH: fast length adjustment of short reads to improve genome assemblies. Bioinformatics, 27, 2957-63.

Malmstrøm M, Matschiner M, Tørresen OK et al. (2016) Evolution of the immune system influences speciation rates in teleost fishes. Nature Genetics, $\mathbf{4 8 .}$

Mayrose I, Zhan SH, Rothfels CJ et al. (2011) Recently Formed Polyploid Plants Diversify at Lower Rates. , 333.

Meyer M, Kircher M (2010) Illumina sequencing library preparation for highly multiplexed target capture and sequencing. Cold Spring Harbor protocols, 2010, doi:10.1101/pdb.prot5448.

Mirarab S, Warnow T (2015) ASTRAL-II : coalescent-based species tree estimation with many hundreds of taxa and thousands of genes. Bioinformatics, 45, 44-52.

Paradis E, Claude J, Strimmer K (2018) APE : Analyses of Phylogenetics and Evolution in R language. , 20, 289-290.

Phuong M, Mahardika G (2017) Targeted sequencing of venom genes from cone snail genomes reveals coupling between dietary breadth and venom diversity. biorxiv.

Phuong MA, Mahardika GN (2018) Targeted Sequencing of Venom Genes from Cone Snail 
Genomes Improves Understanding of Conotoxin Molecular Evolution. , 1-15.

1008

1009

1010

1011

1012

1013

1014

1015

1016

1017

1018

1019

1020

1021

1022

1023

1024

1025

1026

1027

1028

1029
Phuong MA, Mahardika GN, Alfaro ME (2016) Dietary breadth is positively correlated with venom complexity in cone snails. BMC Genomics, $\mathbf{1 7}, 401$.

Pigliucci M (2008) Is evolvability evolvable? Nature reviews. Genetics, 9, 75-82.

Puillandre N, Bouchet P, Duda TF et al. (2014a) Molecular phylogeny and evolution of the cone snails (Gastropoda, Conoidea). Molecular phylogenetics and evolution, 78, 290-303.

Puillandre N, Duda TF, Meyer C, Olivera BM, Bouchet P (2014b) One, four or 100 genera? A new classification of the cone snails. Journal of Molluscan Studies, 1-23.

Puillandre N, Kantor YI, Sysoev a. et al. (2011) The dragon tamed? A molecular phylogeny of the Conoidea (Gastropoda). Journal of Molluscan Studies, 77, 259-272.

Puillandre N, Tenorio MJ (2018) Molluscan Studies. , 200-210.

Pyron RA, Burbrink FT (2011) EXTINCTION , ECOLOGICAL OPPORTUNITY , AND THE ORIGINS OF GLOBAL SNAKE DIVERSITY. , 163-178.

Rabosky DL (2014) Automatic detection of key innovations, rate shifts, and diversitydependence on phylogenetic trees. PloS one, 9, e89543.

Rabosky DL, Goldberg EE (2017) FiSSE : A simple nonparametric test for the effects of a binary character on lineage diversification rates. , 1432-1442.

Rabosky DL, Grundler M, Anderson C et al. (2014) BAMMtools : an R package for the analysis of evolutionary dynamics on phylogenetic trees. , 701-707.

Rabosky DL, Huang H (2016) A Robust Semi-Parametric Test for Detecting Trait-Dependent Diversification. Systematic biology, 65, 181-193.

Rabosky DL, Santini F, Eastman J et al. (2013) Rates of speciation and morphological evolution are correlated across the largest vertebrate radiation. Nature communications, 4, 1-8. 
1030 Robertson FM, Gundappa MK, Grammes F et al. (2017) Lineage-specific rediploidization is a

1031

1032

1033

1034

1035

1036

1037

1038

1039

1040

1041

1042

1043

1044

1045

1046

1047

1048

1049

1050

1051

1052 mechanism to explain time-lags between genome duplication and evolutionary diversification. , 1-14.

Ruby JG, Bellare P, Derisi JL (2013) PRICE: software for the targeted assembly of components of (Meta) genomic sequence data. G3, 3, 865-80.

Safavi-Hemami H, Gajewiak J, Karanth S et al. (2015) Specialized insulin is used for chemical warfare by fish-hunting cone snails. Proceedings of the National Academy of Sciences, 112, $1743-1748$.

Santini F, Harmon LJ, Carnevale G, Alfaro ME (2009) Did genome duplication drive the origin of teleosts? A comparative study of diversification in ray-finned fishes. BMC evolutionary biology, 9, 194.

Sayyari E, Mirarab S (2017) Fast Coalescent-Based Computation of Local Branch Support from Quartet Frequencies Article Fast Track. , 33, 1654-1668.

Schmieder R, Edwards R (2011) Quality control and preprocessing of metagenomic datasets. Bioinformatics, 27, 863-864.

Simakov O, Marletaz F, Cho S-J et al. (2013) Insights into bilaterian evolution from three spiralian genomes. Nature, 493, 526-31.

Slater GSC, Birney E (2005) Automated generation of heuristics for biological sequence comparison. BMC bioinformatics, $\mathbf{6}, 31$.

Smit AFA, Hubley R, Green P (2015) RepeatMasker Open-4.0.

Soltis DE, Albert V a, Leebens-Mack J et al. (2009) Polyploidy and angiosperm diversification. American journal of botany, 96, 336-48.

Stamatakis A (2006) RAxML-VI-HPC: Maximum likelihood-based phylogenetic analyses with 
thousands of taxa and mixed models. Bioinformatics, 22, 2688-2690.

1054 Sunagar K, Casewell NR, Varma S et al. (2016) Deadly Innovations: Unraveling the Molecular

1055 Evolution of Animal Venoms. , 1-27.

1056 Tank DC, Eastman JM, Pennell MW et al. (2015) Nested radiations and the pulse of angiosperm

1057 diversification : increased diversification rates often follow whole genome duplications.

1058 Uribe JE, Puillandre N, Zardoya R (2017) Phylogenetic relationships of Conidae based on

1059 complete mitochondrial genomes. Molecular Phylogenetics and Evolution, 107, 142-151.

1060 Wagner GP, Altenberg L (1996) Perspective: Complex Adaptations and the Evolution of

1061 Evolvability. Evolution, 50, 967-976.

1062 Wong ESW, Belov K (2012) Venom evolution through gene duplications. Gene, 496, 1-7.

1063 Worms Editorial Board (2017) World Register of Marine Species.

1064 Wu Y, Wang L, Zhou M et al. (2013) Molecular evolution and diversity of Conus peptide toxins, 1065 as revealed by gene structure and intron sequence analyses. PloS one, $\mathbf{8}$, e82495.

1066 Yang AS (2001) Modularity, evolvability, and adaptive radiations: a comparison of the hemi-

1067 and holometabolous insects. Evolution \& development, 3, 59-72.

1068 Yang Z (2007) PAML 4: phylogenetic analysis by maximum likelihood. Molecular biology and 1069 evolution, 24, 1586-91.

1070 Zhan SH, Glick L, Tsigenopoulos CS, Otto SP, Mayrose I (2014) Comparative analysis reveals 1071 that polyploidy does not decelerate diversification in fish. , 27, 391-403. 
bioRxiv preprint doi: https://doi.org/10.1101/359976; this version posted July 1 2018. The copyright holder for this preprint (which was not certified by peer review) is the author/funder, who has granted bioRxiv a license to display the preprint in perpetuity. It is made available under aCC-BY-NC 4.0 International license.

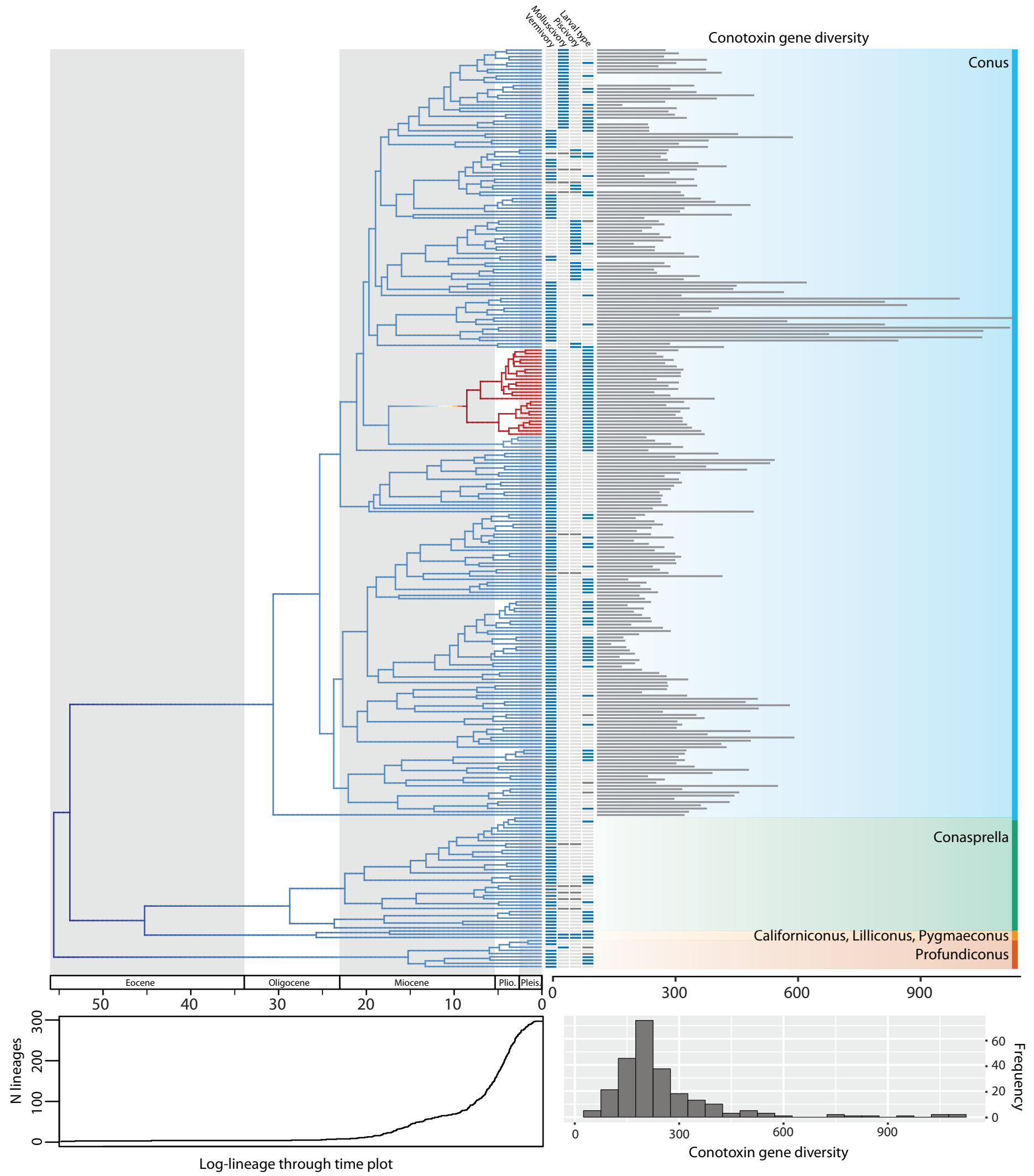

